\title{
L'homme-machine, une esthétique de la modernité ou de son aversion
}

"L'homme-machine", an aesthetic of modernity or of its aversion

\section{Florent Le Bot}

\section{(2) OpenEdition}

\section{Journals}

Édition électronique

URL : http://journals.openedition.org/artefact/3036

DOI : $10.4000 /$ artefact.3036

ISSN : 2606-9245

Éditeur :

Association Artefact. Techniques histoire et sciences humaines, Presses universitaires du Midi

Édition imprimée

Date de publication : 15 mars 2019

Pagination : 143-151

ISBN : 978-2-8107-0623-5

ISSN : 2273-0753

Référence électronique

Florent Le Bot, «L'homme-machine, une esthétique de la modernité ou de son aversion », Artefact [En ligne], 9 | 2018, mis en ligne le 04 mars 2020, consulté le 28 novembre 2020. URL : http:// journals.openedition.org/artefact/3036 ; DOI : https://doi.org/10.4000/artefact.3036

\section{(c)}

Artefact, Techniques, histoire et sciences humaines est mise à disposition selon les termes de la Licence Creative Commons Attribution - Pas d'Utilisation Commerciale - Pas de Modification 4.0 International. 


\section{L'homme-machine, une esthétique de la modernité ou de son aversion}

\section{Florent Le Bot}

\section{Résumé}

Dans ce dossier, nous interrogeons le théâtre et l'iconographie (avec un détour par la littérature) dans l'entre-deux-guerres, pour prendre la mesure des enjeux traversant les différentes représentations de l'Homme confronté à la machine. Cristina Trinchero dans "Machines théâtrales et hommes-machine sur les planches » compare le théâtre futuriste au théâtre de l'existence de Jean-Victor Pellerin. La crise de modernité, qui nourrit ces textes et s'en alimente, trouve également écho dans une certaine iconographie de l'époque, véritable allégorie sociale. Max Bonhomme analyse les images de presse des années 1930 au prisme des discours idéologiques, politiques et sociologisant d'un certain nombre d'essais et de pamphlets contemporains. Les deux articles permettent de croiser des débats qui, au-delà du machinisme, touchent à l'organisation du travail, à l'ordre social, économique et politique, et à la représentation de la vie en société.

\section{Mots-clés}

crise, iconographie, machine, mécanisation, modernité, rationalisation, travail, théâtre

95 Florent Le Bot, « L'homme-machine, une esthétique de la modernité ou de son aversion », Artefact, 9, 2018, p. 143-151. 


\section{"L'homme-machine", an aesthetic of modernity or of its aversion}

\section{Abstract}

In this file, we examine the theater and the iconography (with a detour by the literature) in the inter-war period, to take the measure of the stakes crossing the different representations of the Man confronted with the machine. Cristina Trinchero in "Theatrical machines and machine-men on the boards" compares the futuristic theater to the theater of the existence of Jean-Victor Pellerin. The crisis of modernity that feeds these texts, is also echoed in a certain iconography of the time, a real social allegory. Max Bonhomme analyzes the press images of the 1930s in the prism of the ideological, political and sociologizing discourses of a number of contemporary essays and pamphlets. The two articles make it possible to cross debates which, beyond mechanization, affect the organization of work, the social, economic and political order, and the representation of life in society.

\section{Keywords}

crisis, iconography, machine, mechanization, modernity, rationalization, work, theater 


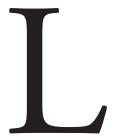
a distance et l'écart entre l'Homme ${ }^{1}$ et la machine sont un sujet ancien dont se sont emparés les philosophes du XviI ${ }^{\mathrm{e}}$ siècle (Descartes, Leibniz, etc.), du XviII ${ }^{\mathrm{e}}$ siècle (Diderot, La Mettrie ${ }^{2}$, etc.) et les penseurs de l'époque contemporaine (Auguste Comte, Jacques Ellul, etc.). Il recouvre trois enjeux au moins : celui de la différence de l'homme par rapport à l'animal et de l'écart entre l'homme et son Créateur ; celui plus pragmatique de la connaissance de la physiologie humaine, d'une part, et de celle de la mécanique des objets terrestres et célestes, d'autre part ; l'enjeu enfin, de la représentation par les artistes, de toutes disciplines, des deux dimensions précédentes, soit la part existentielle et la part fonctionnelle. Les hommes sont-ils des machines comme les autres? Qu'empruntent les machines aux hommes ? Enfin, les machines sont-elles susceptibles de discipliner, de dominer, voire de remplacer l'homme ? Cette manifestation évidente d'intranquillité trouve ses sources dans le $\mathrm{XIX}^{\mathrm{e}}$ siècle industrialiste et ses expressions technophobes (ainsi du mythe du soulèvement des machines) $)^{3}$, mais résonne particulièrement durant le sombre $\mathrm{xx}^{\mathrm{e}}$ siècle, celui des deux guerres mondiales, des crises économiques, des génocides, des bombes atomiques et des accidents nucléaires, et celui du désastre écologique. Les années 1920-1930, au cours desquelles coexistent l'espoir d'une libération de l'homme par la machine ${ }^{4}$ et la prophétie d'une disparition de l'humanité du fait de son hubris mécaniste, marquent une étape dans l'entremêlement de ces thèmes.

Ainsi, dans ce dossier, nous interrogeons singulièrement le théâtre et l'iconographie (avec un détour par la littérature) dans l'entre-deux-guerres, pour prendre la mesure des enjeux existentiels et fonctionnels traversant les différentes représentations des hommes et des femmes confrontés à la machine. Derrière ces questions se cachent celles de l'avenir de l'homme et de la civilisation qui résonnent particulièrement dans les années 1920,

\footnotetext{
1. L'Homme est ici envisagé comme une représentation essentialisée des humains.

2. Julien Offroy de La Mettrie, L’Homme Machine, Paris, Folio Gallimard, 1999 ( $1^{\text {re }}$ éd. 1747).

3. Annie Amartin-Serin, La Création défiée. L’homme fabriqué dans la littérature, Paris, PUF, 1996 et François Jarrige, Techno-critiques. Du refus des machines à la contestation des technosciences, Paris, La Découverte, 2014.

4. Notamment l'économiste Jacques Duboin qui, dans les années 1930, prophétise l'ère de l'abondance grâce aux machines. Olivier DARD, "Sortir de la crise par la technique et par la science ? Les réponses de l'abondancisme de Jacques Duboin ", dossier "Les milles peaux du capitalisme " coordonné par Olivier Dard, Claude Didry, Florent Le Bot et Cédric Perrin, L'Homme et la société, no 195-196, 2015-1, p. 127-148.
} 
à la suite de la Grande Guerre - introduction à grande échelle de la guerre mécanisée -, et encore avec la crise généralisée des années 1930. Cristina Trinchero, dans "Machines théâtrales et hommes-machine sur les planches ", compare le théâtre futuriste de Filippo Tommaso Marinetti et de ses épigones (Luciano Folgore, Enrico Prampolini, etc.) au théâtre de l'existence de Jean-Victor Pellerin. Un tableau contrasté de la présence au monde des machines s'observe aussi bien chez le dramaturge français, à travers sa quête d'une vie authentique, que chez les "Modernolâtres " italiens a priori plus technophiles, mais chez qui surgissent malgré tout des notes de pessimisme face au constat de l'emprise machinique. La crise de modernité qui nourrit ces textes et s'en alimente, trouve également écho dans une certaine iconographie de l'époque, véritable allégorie sociale. Max Bonhomme analyse les images de presse des années 1930 (celles publiées dans Arbeiter Illustrierte Zeitung, Les Temps nouveaux, Life et VU) au prisme des discours idéologiques, politiques et sociologisants d'un certain nombre d'essais et de pamphlets contemporains ${ }^{5}$. L'imagerie du déclin civilisationnel y cohabite avec une vision plus lumineuse du devenir de l'humanité. Les deux articles permettent de croiser des débats qui, au-delà 146 du machinisme, touchent à l'organisation du travail, à l'ordre social, économique et politique, et à la représentation de la vie en société.

\section{La mesure entre l'homme de Vitruve et «l'ouvrier crucifié »}

La machine et le robot (dont le nom a été introduit en 1920 par le tchèque Karel Čapek dans la pièce de théâtre Rossum's Universal Robots) mettent en cause le paradigme humaniste de "l'homme mesure de toutes choses". Dans un parallèle saisissant Max Bonhomme dévoile le glissement opéré de l'homme de Vitruve, célèbre dessin de Léonard de Vinci dans lequel les proportions parfaites du corps humain s'inscrivent dans un cercle et un carré, mesure de la création divine, jusqu'à l'ouvrier crucifié sur une roue dentée du peintre František Kupka qui présente un ouvrier ligoté à un engrenage, réduit à n'être qu'un simple rouage dans la machine industrielle, sacrifié comme le Christ, ici sur l'autel du capitalisme. Avec la question du rythme de la machine, de sa puissance et de la capacité que l'homme a, ou non, de

5. Notamment Georges Duhamel, Scènes de la vie future, Paris, Mille et une nuits, 2003 (1930). 
la contrôler, intervient l'idée de mesure : la mesure comme affirmation de tempérance contre l'hubris prométhéenne. Mesure et démesure sont d'ailleurs illustrées par l'usage que certains graphistes et photographes font de la juxtaposition de machines géantes ou d'ouvrages monumentaux à côté de figures humaines réduites à leurs plus humbles proportions, tandis que, pour les communistes, le gigantisme de la machine est rapporté à celui du travailleur lui-même dans une perspective de valorisation. Ce champ de la mesure se décline par analogie avec ce qui constituerait l'identité de pays « démesurés", dans tous les sens du terme, comme les États-Unis et l'URSS, deux géants technologiques, versus la France présentée, dans une tradition toute cartésienne, comme " pays de la mesure » et de l'équilibre ${ }^{6}$.

La question posée est d'abord celle de l'autonomie de l'homme par rapport à la machine. Le futurisme et le théâtre de l'existence s'emparent du sujet. La prise du contrôle par la machine se lit dans le futurisme comme une entreprise à célébrer, cette dernière faisant ce que l'homme, son constructeur, ne saurait pas faire seul. La machine, dans une configuration quasiment transhumaniste, y est présentée comme une prothèse artificielle augmentant l'être humain ${ }^{7}$. Chez Jean-Victor Pellerin, la dimension de routine ("des individus presque robotisés ») et de dépersonnalisation l'emporte, à travers un processus inexorable de déshumanisation. La cadence machinique répétitive met en péril le rythme même de nos vies jusqu’à l'épuisement ${ }^{8}$. Pour le dramaturge, il s'agit « de fuir la dimension trop matérielle d'une vie automatisée à ce point et de rattraper sa dimension authentique».

\section{Vers la cité industrielle parfaite ?}

L'organisation scientifique du travail intervient en arrière-fond de ces débats. La planification, le chronométrage des tâches et le travail à la

6. À propos de la représentation de longue durée de la France comme pays cartésien, Sudhir Hazareesingh, Ce pays qui aime les idées. Histoire d'une passion française, Paris, Flammarion, 2015. 7. Florent Le Bot, Olivier Dard, Claude Didry, Camille Dupuy et Cédric Perrin (dir.), dossier "L'homme machine II. Du travailleur augmenté à l'homme augmenté ", L'Homme et la société, $n^{\circ} 207,2018-2$.

8. Il serait intéressant de relire ce texte à la lumière de ce qu'écrit le sociologue Harmut Rosa s'agissant de l'accélération du temps dans le contexte de la modernité : accélération technique, accélération du rythme de vie et accélération des transformations sociales et culturelles. Hartmut RosA, Accélération. Une critique sociale du temps, Paris, La Découverte, 2010 (Berlin, 2008). 
chaîne sont les trois emblèmes d'une rationalisation de la production bien plus complexe et plus diversifiée dans les faits, et qui laisse penser à certains que le travail échappe aux mains de l'homme. Max Bonhomme cite ainsi Georges Duhamel qui, en 1933, " défend, contre les excès de la rationalisation technique, la transmission des savoirs manuels et la connaissance intuitive que peut avoir un praticien de son outil de travail, qui suppose de s'inscrire dans le temps long de l'apprentissage ". Jean Giono tient des propos comparables dans la Lettre aux paysans sur la pauvreté et la paix, dans laquelle il dénonce les temps nouveaux de l'usine et de ses sirènes (dans les deux sens du terme), de la civilisation technique et de l'aliénation de l'homme, assimilés à la guerre :

La transformation que vous subissez [les paysans], l'artisan l'a subie totalement. Il a perdu sa qualité artisanale ; il est devenu un ouvrier. Il a perdu tout ce à quoi vous essayez de vous raccrocher : la vie, la paix et la liberté. Je vous ai cent fois raconté la vie de mon père. C'était un artisan cordonnier. Il savait faire une paire de souliers depuis le rouleau de cuir jusqu'aux lacets. [...] Il était entièrement maître de sa vie ; comme un homme digne de ce nom doit être. [...] Il a chanté pendant toute sa vie, jusqu’à la guerre. Cet artisan cordonnier est devenu un ouvrier cordonnier. Il travaille chez Bata. Il sait coudre une trépointe9. L'ouvrier de Bata met à peine une demi-heure. Il y est plus habile que mon père mais il ne sait faire que ça. Il ne sait pas monter tout le soulier. Il coud sa trépointe et il passe le travail à un autre. Malheureusement pour lui personne au monde n'a besoin d'une trépointe; on a besoin de souliers finis. L'ouvrier ne peut quitter sa chaise chez Bata. S'il s'en allait de là il ne pourrait pas vivre. Il n'a plus un métier qui le fait vivre n’importe où. Il ne peut plus vivre qu'intercalé à la place des trépointes dans l'ordre Bata. Sous peine de mourir il ne peut ni se déplacer, ni vivre (car vivre est autre chose que de coudre des trépointes). Il est obligé de rester là ; il faut qu'il s'y oblige physiquement. Il est prisonnier et sa famille est prisonnière. Et, si on lui donne quinze jours de congés payés par an, je dis qu’à côté

9. Bande de cuir souple profilée, fixée autour de la chaussure, qui sert à faire la liaison entre la semelle première et la tige, d'une part, et le semelage, d'autre part. 
des grandes vacances perpétuelles de mon père ce qu’on appelle ici progrès n'est qu'une sérieuse régression ${ }^{10}$.

Giono s'inscrit ici dans une controverse qui oppose durant les années 1930 bataphiles et bataphobes. Les milieux organisateurs vantent les réalisations en matière de rationalisation de la production et de la distribution de cette entreprise tchèque qui, à partir d'une ville-usine, a essaimé durant l'entre-deux-guerres dans le monde entier. Ils sont rejoints à ce propos par des architectes (Le Corbusier), des écrivains (Paul Claudel), des politiques (Jacques Doriot) pour qui Bata constitue une étape sur la voie de la cité industrielle parfaite. Les concurrents de Bata dans toute l'Europe en dénoncent le gigantisme et les méthodes jugées " déloyales ». Cela débouche notamment en France sur une loi anti-Bata en 1936 qui bloque durablement l'extension de l'entreprise ${ }^{11}$.

Les textes et les images présentés et analysés par Cristina Trinchero et Max Bonhomme s'inscrivent ainsi dans des débats plus larges, tandis que l'enjeu du rapport de l'homme à la machine offre l'occasion de polémiques autour de l'industrialisation, de l'exode rural et de l'urbanisation, de l'urbanisme et de l'architecture (les gratte-ciel comme modèles ou repoussoirs ${ }^{12}$ ), de la démographie et de la peur de la dénatalité, de la société de consommation et du modèle (ou contre-modèle) américain ${ }^{13}$, etc.

10. Jean Giono, Lettre aux paysans sur la pauvreté et la paix, Paris, éd. de la Butte aux Cailles, 1983 (Grasset, 1938), p. 70-73. Florent Le Bot, «Dans l'atelier du cordonnier. Guéhenno, Giono, Guilloux, artisans pacifistes », Jeanyves Guérin, Jean-Kely Paulhan et Jean-Pierre Rioux (dir.), Jean Guéhenno : guerres et paix, Villeneuve-d'Ascq, Presses universitaires du Septentrion, 2009, p. 105-118.

11. Florent Le Вот, "Bata and the modernity. A French debate during the Inter War years", for Company Towns of the Bata Concern, international conference, 24-25. March 2011, Prague, (République Tchèque), non publié. Florent Le Bot, "La "Famille" du cuir contre Bata. Malthusianisme, protectionnisme, xénophobie et antisémitisme dans le monde de la chaussure en France, 1930-1950" Revue d'histoire moderne et contemporaine, n 52-4, octobre-décembre 2005, p. 131-151.

12. Jean-Louis Cohen (dir.), Scènes de la vie future : l'architecture européenne et la tentation de l'Amérique, 1893-1960, Paris/Montréal, Flammarion/Centre canadien d'architecture, 1995.

13. Philippe Roger, L'ennemi américain. Généalogie de l'antiaméricanisme français, Paris, Seuil, 2002 ; Olivier DARD, Hans-Jürgen LüSEBrink (éd.), Américanisations et antiaméricanismes comparés, Villeneuve-d'Ascq, Presses universitaires du Septentrion, 2008. 


\section{L'humanité perdue ou le déclin de la civilisation}

La question des machines est finalement très politique. Un débat qui traverse les démocraties entre croyance dans le progrès économique et social par la mécanisation et hantise du déclin, voire de la destruction, de la civilisation submergée par le machinisme ${ }^{14}$. Un débat qui oppose également démocraties et régimes communistes et fascistes à l'aune de l'Homme nouveau $^{15}$. Cette figure de l'homme amplifié, métamorphosée par la volonté politique, avec l'appui de la force technique, est présente en filigrane dans le texte de Max Bonhomme, qui y analyse le productivisme communiste. Elle permet aussi de mettre en perspective l'article de Cristina Trinchero à travers les relations ambivalentes, mais nombreuses, entre futurisme et fascisme $^{16}$. Enfin, il y a la question de la guerre fortement corrélée à la mécanisation et dont s'empare, parmi les plus pessimistes, Georges Bernanos :

[Des malheureux] aimeront mieux tuer que penser, voilà le malheur ! Et justement vous les fournissez de mécaniques! La mécanique est faite pour eux. En attendant la machine à penser qu'ils attendent, qu'ils exigent, qui va venir, ils se contenteront très bien de la machine à tuer, elle leur va même comme un gant. Nous avons industrialisé la guerre pour la mettre à leur portée. Elle est à leur portée, en effet ${ }^{17}$.

Il resterait à observer la manière dont ces questions rebondissent avec les années de croissance économique dites des "Trente Glorieuses ". Pensons à Jean Fourastié et aux espoirs mis dans la technique revisitée par

14. Sur ce dernier aspect, René Barjavel, Ravage, Paris, Denoël, 1943 et Georges Bernanos, La France contre les robots, Bègles, Le castor astral, 2015 (1947). Olivier DARD, " "L'homme des machines" de Georges Bernanos ", Florent Le Bot, Olivier Dard, Claude Didry, Camille Dupuy et Cédric Perrin (dir.), dossier "L'homme machine I. Le travailleur-machine ", L'Homme et la société, $\mathrm{n}^{\circ} 205,2017-3$.

15. Marie-Anne Matard-Bonucci et Pierre Milza, L'Homme nouveau dans l'Europe fasciste, 19221945. Entre dictature et totalitarisme, Paris, Fayard, 2004.

16. Giovanni Lista (éd.), Marinetti et le futurisme, Lausanne, L'Âge d'Homme, 1977 ; Éric Michaud, "Le présent du futurisme. Les vertiges de l'auto-destruction ", Mil neuf cent. Revue d'histoire intellectuelle, 2003-1, n 21, p. 21-42.

17. Georges Bernanos, Les grands cimetières sous la lune, Paris, Points, 2014 [1938], p. 22. 
la productivité ${ }^{18}$ ou encore, pour l'iconographie, aux œuvres de Fernand Léger évoquées par Max Bonhomme. Le sujet, déjà bien exploré, reste vaste.

\section{Lauteur}

Florent Le Bot est enseignant à l'université d'Évry Val d'Essonne - université Paris-Saclay et chercheur à l'IDHES Évry. Ses travaux portent en particulier sur l'histoire des entreprises au xx $x^{\mathrm{e}}$ siècle et il a notamment publié, avec Cédric Perrin et Fabrice Grenard, Histoire économique de Vichy. L'État, les hommes, les entreprises, Paris, Perrin, 2017, et a codirigé deux dossiers sur le thème de l'homme-machine pour la revue L'Homme et la société (n²05, 2017-3; n²07, 2018-2). Contact : florent.lebot@univ-evry.fr ; https://univ-evry.academia.edu/FlorentLeBot.

18. Jean FourastiÉ, Le Grand espoir du XXe siècle, Progrès technique, progrès économique, progrès social, Paris, PUF, 1949. Régis Boulat, Jean Fourastié, un expert en productivité. La modernisation de la France (années trente-années cinquante), Besançon, Presses universitaires de Franche-Comté, 2008. 